\title{
Análisis de la tecnología lítica del sitio Laguna La Larga (partido de Adolfo Gonzales Chaves, provincia de Buenos Aires)
}

\author{
María José Colantonio \\ Recibido 05 de mayo 2021. Aceptado 13 de septiembre 2021
}

\begin{abstract}
RESUMEN
En este trabajo se presentan y discuten los resultados del análisis de la tecnología lítica del sitio Laguna La Larga (partido de Adolfo Gonzales Chaves, Buenos Aires). Los objetivos principales son interpretar los modos de abastecimiento y las técnicas de talla de las diferentes materias primas y determinar las trayectorias de producción y las estrategias tecnológicas empleadas por las poblaciones cazadorasrecolectoras que ocuparon la laguna durante el Holoceno tardío. Con este propósito, se efectuó el análisis tecnomorfológico de los materiales líticos y la reconstrucción de las cadenas operativas para cada roca. Se registró una amplia variabilidad de materias primas líticas, siendo la ortocuarcita del Grupo Sierras Bayas y la ftanita las rocas mayormente explotadas. En cuanto a las cadenas operativas, se evidencia la presencia mayoritaria de etapas de manufacturas intermedias y finales sobre las rocas, la estandarización de formas base en ortocuarcita, entre las cuales se seleccionaron lascas grandes con módulos alargados para confeccionar filos largos, y se obtuvieron lascas pequeñas y anchas no estandarizadas sobre otras rocas (e.g., raspadores pequeños en ftanita).
\end{abstract}

Palabras clave: Cazadores-recolectores; Técnicas de talla; Cadenas operativas; Área Interserrana; Holoceno tardío.

\section{Analysis of the lithic technology of the Laguna La Larga site (Adolfo Gonzales Chaves district, Buenos Aires province)}

\begin{abstract}
In this paper, the results of the lithic technology analysis of the Laguna La Larga archaeological site (Adolfo Gonzales Chaves, Buenos Aires) are presented and discussed. The main objectives are to interpret the procurement modes and the knapping techniques of each lithic raw material and to determine the technological strategies involved and the trajectories of production developed by hunter-gatherer groups that occupied the lake during the Late Holocene. A techno-morphological analysis of the lithic materials and the reconstruction of the chaines opératoires for each rock were carried out. A wide variability of lithic raw materials was recorded, with orthoquartzite from the Sierras Bayas Group and chert the most exploited. Regarding the chaînes opératoires, there is evidence of the dominance of intermediate and final manufacturing stages, the standardization of orthoquartzite blanks, where large flakes with elongated sections were selected to make long edges (side-scrapers and knives), and the obtaining of small, wide, and non-standardized flakes from other rocks (e.g., small end-scrapers in chert).
\end{abstract}

Keywords: Hunter-gatherers; Knapping techniques; Chaînes opératoires; Interhill area; Late Holocene.

María José Colantonio. Facultad de Ciencias Sociales, Universidad Nacional del Centro de la Provincia de Buenos Aires (UNICEN), Argentina. Avenida Del Valle 5737 (7400), Olavarría, Buenos Aires, Argentina. E-mail: tresajose2002@yahoo.com.ar

Intersecciones en Antropología 22(2), julio-diciembre: 223-235. 2021. ISSN-e 1850-373X

https://doi.org/10.37176/iea.22.2.2021.632

Facultad de Ciencias Sociales - UNICEN - Argentina 


\section{INTRODUCCIÓN}

En la subregión Pampa Húmeda, las materias primas líticas aptas para la talla han sido caracterizadas como un recurso localizado, restringido y distribuido de forma heterogénea en el paisaje; lo cual ha permitido plantear la utilización de diversas estrategias tecnológicas vinculadas al abastecimiento, circulación, procesos de talla y uso de las rocas por parte de los grupos cazadores-recolectores durante el Holoceno (e.g., Martínez y Mackie, 20032004; Bayón et al., 2006; Massigoge y Pal, 2011; Messineo, 2011). Una multiplicidad de factores como, por ejemplo, los circuitos de movilidad de los grupos cazadores-recolectores, las restricciones sociales en el acceso a las canteras, los objetivos de talla, las distancias a las fuentes de materias primas, la disponibilidad y calidad de las rocas, han influido en las estrategias de aprovisionamiento y traslado de estas (Martínez y Mackie, 2003-2004; Bayón y Flegenheimer, 2004; Bonomo, 2005; Bayón et al., 2006; Messineo, 2008, entre otros).

Básicamente, se plantearon dos maneras de abastecimiento que facilitan la circulación de los recursos hacia áreas alejadas de las fuentes: una de forma directa, que implica la obtención de las rocas en sus fuentes de origen por parte de las poblaciones humanas; y la otra indirectamente, a través de redes de interacción entre los grupos cazadoresrecolectores (Martínez y Mackie, 2003-2004; Bayón y Flegenheimer, 2004; Mazzanti, 2006; Colombo, 2011; entre otros). El abastecimiento directo de los recursos líticos puede darse de dos maneras posibles: por un lado, como una tarea secundaria dentro de otras actividades de subsistencia (embedded strategies, Binford, 1979) y, por otro, mediante viajes especiales realizados por partidas logísticas a las zonas de abastecimiento (direct strategies, Gould y Saggers, 1985).

Para el Holoceno tardío, la materia prima principalmente explotada fue la ortocuarcita del Grupo Sierras Bayas (GSB). Si bien esta tendencia predominó en diversos contextos arqueológicos de la subregión Pampa Húmeda, también se registró en algunos sectores puntuales un uso preferencial de rocas locales, como la ftanita en el extremo noroccidental de Tandilia (Messineo, 2011). En cuanto a la forma de adquirir estos recursos, los grupos humanos utilizaron básicamente dos estrategias complementarias: el aprovisionamiento de los individuos (Khun, 1994), a partir del cual trasladaban las rocas como parte de su toolkit (estrategia implementada durante todo el Holoceno), y el aprovisionamiento de los lugares, que implicó la acumulación intencional de rocas en ciertos puntos del paisaje donde estas eran escasas o nulas. Esta última estrategia fue registrada para el Holoceno tardío en la cuenca media del río Quequén Grande (Martínez y Mackie, 2003-2004) y en sectores próximos al litoral atlántico (Bayón et al., 2006).

Particularmente para el río Quequén Salado, las investigaciones arqueológicas permitieron discutir temas vinculados con la variabilidad del registro arqueológico, la dinámica de movilidad de los grupos humanos entre la costa y el interior, la distribución diferencial de las materias primas y las estrategias tecnológicas implementadas en la confección de los artefactos líticos, entre otros (Madrid et al., 2002). Bajo este contexto y para contribuir con las temáticas anteriormente mencionadas, en este trabajo se llevó a cabo el análisis tecnomorfológico y de cadenas operativas de los artefactos líticos procedentes del sitio Laguna La Larga (en adelante, LLA), ubicado en las inmediaciones del curso superior del río Quequén Salado (Figura 1a). El objetivo principal es determinar las prácticas tecnológicas vinculadas con los modos de adquisición y explotación de las rocas, las actividades de talla y las trayectorias de producción de las diferentes materias primas líticas utilizadas por los cazadores-recolectores que ocuparon la laguna durante el Holoceno tardío. La información generada será comparada con los resultados obtenidos en otros contextos arqueológicos de la cuenca del río Quequén Salado y de cuencas cercanas, con el fin de indagar sobre las tendencias en el abastecimiento, traslado y producción de las rocas a nivel microrregional y para el área Interserrana durante el mismo período.

\section{ANTECEDENTES}

\section{Base de recursos líticos de la subregión Pampa Húmeda}

Las fuentes de abastecimiento lítico se encuentran ubicadas en cuatro zonas específicas de la subregión: las sierras de Tandilia y Ventania, el área Interserrana y el litoral atlántico (Figura 1b). Respecto de las primeras, se registraron en el sector centro-sur ortocuarcita GSB, sílices y dolomía silicificada (a $120 \mathrm{~km}$ al noreste de LLA) (Flegenheimer et al., 1996; Colombo, 2011); en el sector sudoriental, ortocuarcita de la Formación Balcarce y cuarzo (a $200 \mathrm{~km}$ al este de LLA) (Bonnat, 2021); y en el 


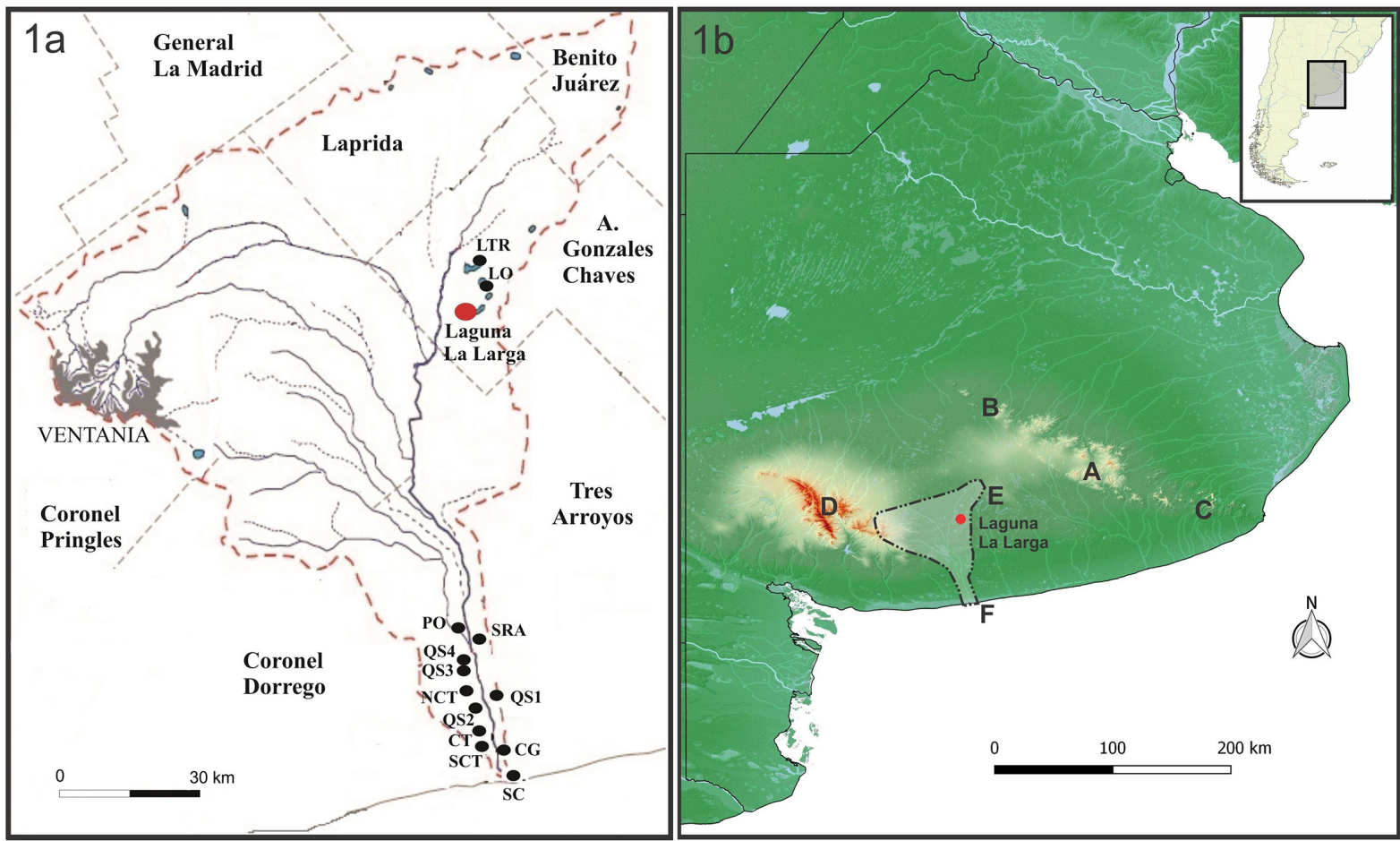

Referencias: LTR (Laguna Tres Reyes); LO (Laguna Ovalla); SC (Sitio Caracolero); CG (Campo Girasoles); SCT (Sur Cueva del Tigre); CT (Cueva del Tigre); QS1 (Quequén Salado 1); QS2 (Quequén Salado 2); QS3 (Quequén Salado 3); QS4 (Quequén Salado 4); NCT (Norte Cueva del Tigre); SRA (Sur Represa Antigua); PO (Puente Oriente); A (sector centro sur del sistema serrano de Tandilia); B (sector noroccidental de Tandilia; C (sector oriental de Tandilia); D (Sistema Serrano de Ventania); E (Afloramientos de Adolfo Gonzales Chaves) y F (Depósitos de la costa atlántica).

Figura 1. Ubicación de los sitios arqueológicos en la cuenca del río Quequén Salado (1a) y localización de las fuentes de materias primas líticas de la subregión Pampa Húmeda (1b).

noroccidental, granito, ftanita, ortocuarcita GSB, dolomía silicificada y cuarzo (a $130 \mathrm{~km}$ al norte de LLA) (Messineo y Barros, 2015). Las ortocuarcitas GSB son rocas sedimentarias de grano medio a fino, las cuales presentan fractura concoide y son de muy buena calidad para la talla. Estas rocas yacen bajo la forma de filones y grandes bloques desprendidos del afloramiento que presentan corteza espesa producto de la meteorización (Flegenheimer et al., 1996). Según Colombo (2011), esta roca se extrajo de los filones, nódulos y clastos de diversos tamaños, los cuales se explotaron para obtener núcleos y lascas nodulares con el fin de ser transportados hacia otros sitios. La ftanita es una roca silícea, muy dura y de grano fino, que presenta fractura concoide y es de muy buena calidad para la talla. Esta roca se presenta bajo la forma de bloques y nódulos de distintas dimensiones. Se ha planteado que su traslado desde las fuentes se produjo bajo la forma de nódulos sin descortezar, núcleos parcialmente descortezados y lascas grandes como formas base (Messineo y Barros, 2015).

En el Sistema Serrano de Ventania se localizaron, en la porción noroccidental, afloramientos primarios y secundarios de ortocuarcitas y granito, y en el sector occidental, riolita y metacuarcita de distintas formaciones (entre 110 y $150 \mathrm{~km}$ al oeste de LLA) (Oliva y Moirano, 1997; Catella, 2014). Las metacuarcitas son rocas sedimentarias silicoclásticas que sufrieron transformaciones por procesos metamórficos. Estas tienen una estructura interna que presenta rajaduras e inclusiones, lo cual hace que su calidad para la talla sea menor en comparación con las ortocuarcitas de Tandilia (Catella, 2014). Por su parte, la riolita es una roca de textura porfírica, con fenocristales de cuarzo y feldespato, cuya calidad para la talla es regular y buena. Para esta roca solo se han explotado los afloramientos primarios que presentan las mejores calidades para la talla (Oliva y Moirano, 1997).

En el área Interserrana, se ubicaron algunos afloramientos aislados de limolita silicificada (a 30 $\mathrm{km}$ al noreste de LLA) (Madrid y Salemme, 1991), areniscas cuarcíticas, cuarcitas de grano grueso y depósitos secundarios de rodados de metacuarcita y de cuarzo (a $100 \mathrm{~km}$ al sudoeste de LLA) (Bayón et al., 2006). Por último, en el litoral atlántico (a $80 \mathrm{~km}$ al sur de LLA), se encuentran disponibles rodados con diámetros de $9 \mathrm{~cm}$ de promedio (algunos pueden llegar a los $15 \mathrm{~cm}$ ) y compuestos 
por una amplia variedad de rocas, como basaltos, sílices y cuarcitas de buena calidad para la talla (Bonomo, 2005).

\section{Investigaciones arqueológicas en la cuenca del río Quequén Salado}

Los primeros trabajos de campo se efectuaron en la década de 1980 en el sector norte de la cuenca e incluyeron prospecciones y excavaciones en la localidad arqueológica Laguna Tres Reyes (Politis y Madrid, 1988; Madrid y Salemme, 1991) y prospecciones y sondeos en las lagunas La Larga, Ovalla y La Sortija (Madrid et al., 2002). Los fechados radiocarbónicos obtenidos en Laguna Tres Reyes permitieron situar las ocupaciones a inicios del Holoceno tardío, entre ca. 2300 y 1800 años AP.

Posteriormente, se llevó a cabo un proyecto orientado al estudio integral del espacio microrregional, con el fin de evaluar el rol que desempeñaron los cursos fluviales transversales a la costa atlántica en la dinámica de los grupos cazadoresrecolectores, desde el Pleistoceno final hasta el Holoceno tardío (Madrid et al., 2002). A partir de prospecciones intensivas en una amplia variedad de geoformas, se detectaron en la cuenca del río Quequén Salado 14 sitios arqueológicos (Figura 1a), algunos en estratigrafía y otros en superficie (Madrid et al., 2002; Hoguin y March, 2008). En el curso inferior se identificaron los sitios Puente Oriente, Sur Represa Antigua, La Represa (3430, 3180, 3050, 2900 y 2110 años AP), Norte Cueva del Tigre, Cueva del Tigre, Quequén Salado 1 (360 y 960 años AP), Quequén Salado 2 (1720 años AP), Quequén Salado 3 y 4 (1240 años AP), Campo de Girasoles y Caracolero. En el curso medio no se registraron sitios, y en el superior se localizaron Laguna Ovalla, Laguna Tres Reyes y LLA. Los sitios con mayor concentración y densidad de hallazgos se hallan en las márgenes de lagunas y cuerpos de agua del curso superior, lo cual señala la alta redundancia ocupacional.

A partir de la información obtenida y teniendo en cuenta las materias primas líticas más explotadas, Barros (2001) observó ciertas tendencias para las zonas lagunares de la cuenca superior del río Quequén Salado. La ortocuarcita GSB fue la roca más utilizada, y se explotaron en menor proporción otras litologías (e.g., rodados costeros, limolita silicificada y riolita). La mayoría de las rocas fueron introducidas en los sitios en un estado avanzado del proceso de manufactura, y se registró la obtención de lascas anchas y laminares, así como la utilización de un modo de débitage unipolar en la confección de los instrumentos. En cuanto a las características del conjunto lítico de los sitios del curso inferior (e.g., Puente Oriente y Cueva del Tigre del Norte), se registraron similitudes con relación al uso de las materias primas registradas para la cuenca superior (Barros, 2001). Posteriormente, Hoguin y March (2008) centraron su interés en el análisis de las cadenas operativas del sitio La Represa, donde se registró un predominio de la ortocuarcita GSB y un bajo porcentaje de corteza, que permitió inferir un desbaste de los núcleos fuera del sitio para estas rocas (March et al., 2011).

Particularmente para LLA ( $38^{\circ} 06^{\prime}$ de latitud sur y $60^{\circ} 31^{\prime}$ de longitud oeste; Figura 1a), se efectuaron prospecciones sistemáticas mediante transectas transversales y perpendiculares en el sector noreste de la laguna, que permitieron detectar cinco secciones de barrancas, donde se recolectaron materiales arqueológicos en superficie (Madrid et al., 2002). En total se recuperaron 634 piezas, 403 de las cuales son artefactos líticos; 165, restos faunísticos; 39, fragmentos cerámicos; 9 , vidrios; 1 , losa; 7, ocres; y 10, ecofactos líticos (Madrid et al., 2002). Los materiales encontrados en estratigrafía $(n=207)$ corresponden a dos sondeos efectuados en la barranca 1, entre los cuales se recuperaron 171 restos faunísticos, 31 artefactos líticos y 5 fragmentos de alfarería (Madrid et al., 2002). A partir del conjunto de fechados obtenidos para la cuenca, de los contextos arqueológicos registrados y de la presencia de ciertos materiales en LLA (e.g., cerámica y puntas de proyectil triangulares pequeñas), el sitio fue situado cronológicamente en el Holoceno tardío.

\section{MATERIALES Y MÉTODOS}

El conjunto lítico analizado en este trabajo es de 402 piezas que provienen de la totalidad de los materiales en estratigrafía del sondeo de la barranca 1 $(\mathrm{n}=31)$ y de una muestra proveniente de superficie $(\mathrm{n}=371)$. El análisis tecnomorfológico y la implementación del modelo de cadena operativa (chaîne opératoire, sensu Leroi-Gourhan, 1943) permitieron determinar las estrategias tecnológicas, los objetivos de talla y las diversas trayectorias de producción implicadas en la explotación de las rocas. Esta herramienta metodológica permite reconstruir la esfera de producción tecnológica a partir del análisis de 
cada una de las etapas encadenadas que transita un artefacto lítico (Lemonnier, 1992).

Desde el punto de vista metodológico, el conjunto lítico fue dividido en grupos según la materia prima y en clases artefactuales (sensu Aschero y Hocsman, 2004). Para lograr una mejor caracterización y reconocimiento de la procedencia de las rocas, se efectuaron comparaciones macroscópicas con muestras de mano pertenecientes a la litoteca del instituto INCUAPA-CONICET y con las descripciones de las rocas de las principales publicaciones para la región pampeana (e.g., Bonomo, 2005; Bayón et al., 2006; Catella, 2014; Carrera Aizpitarte et al., 2015, Messineo y Barros, 2015). En cuanto al análisis de los artefactos formatizados y lascas con filos naturales con rastros complementarios, se siguieron los criterios tecnomorfológicos y los atributos dimensionales planteados por diferentes investigadores (Aschero, 1975, 1983; Aschero y Hocsman, 2004, entre otros). Además, para el análisis de los instrumentos se emplearon criterios tomados de Boëda (1993), Inizan et al. (1995) y Aschero y Hocsman (2004); para los núcleos, los postulados de Paulides (2006); y para los desechos de talla, los criterios propuestos en Bellelli et al. (1985-1987). Los resultados serán presentados siguiendo como eje las materias primas identificadas, en función de entender cómo fue la gestión de cada una de las rocas por parte de los grupos cazadoresrecolectores que ocuparon LLA.

\section{RESULTADOS}

En cuanto a las clases artefactuales, la más representativa está constituida por los desechos de taIla $(72,7 \%)$, seguidos por los instrumentos $(24,1 \%)$, las lascas con filos naturales con rastros complementarios $(2 \%)$ y los núcleos $(1,2 \%)$. Se identificó una amplia variabilidad de materias primas representadas con diferentes frecuencias, entre las que predomina la ortocuarcita GSB $(54,4 \%)$, seguida por la ftanita $(24,4 \%)$, la metacuarcita $(4 \%)$, la limolita silicificada $(3,5 \%) y$, en proporciones ínfimas, sílice, riolita, cuarzo, chert silíceo, dacita, basalto y xilópalo, entre otras (Material suplementario 1).

\section{Ortocuarcita GSB}

El $42,7 \%$ de los instrumentos líticos del sitio fueron confeccionados en esta materia prima (Material suplementario 2; Figura 2). Los soportes mayormente representados son lascas internas $(71,4 \%) y$, en valores inferiores, formas base indeterminables $(14,2 \%)$, lascas externas, lascas internas bipolares y núcleos (4,8\%, cada uno). Entre los grupos tipológicos, se destacan los fragmentos de instrumentos indeterminables $(26,8 \%)$, los instrumentos compuestos (24,4\%; Figura 2A), conformados por la combinación de diferentes tipos de filos (e.g. raederas, raspadores, puntas destacadas, cuchillo y muescas), y las raederas (21,6\%; Figura 2C). En proporciones menores se identificaron raspadores $(9,8 \%)$, muescas, artefactos con retoque sumario, cepillos y cuchillos, entre otros (Material suplementario 2). En lo que respecta al estado, prevalecen los instrumentos fracturados $(57,1 \%)$ por sobre los enteros $(42,9 \%)$. Los tamaños de los instrumentos enteros de ortocuarcita GSB muestran que los medianos pequeños y grandes $(36,8 \%$ cada uno) y medianos grandes $(21,1 \%)$ son los más representativos (Material suplementario 3). En cuanto a los módulos de longitud y anchura, se observó que el mediano alargado está mayormente representado, con un $42,1 \%$, seguido por el corto ancho, con el $21,1 \%$ (Material suplementario 4).

Se registró la extracción de dos tipos de soportes con diferentes fines: por un lado, se encuentran las lascas de tendencia laminar que se utilizaron para la confección de raederas e instrumentos compuestos (Figura 2A y C). Estas fueron extraídas cuidadosamente aplicando una técnica de percusión blanda mediante un golpe tangencial, evidenciado en el registro de talones filiformes con un ángulo de inclinación pronunciado $\left(50^{\circ}\right.$ a $\left.80^{\circ}\right)$, una alta frecuencia de abrasión del talón y un adelgazamiento unifacial de la pieza. Las series técnicas observadas en estos instrumentos fueron retoques extendidos y parcialmente extendidos más microrretoques marginales. La mayoría presenta una tendencia en operaciones de débitage, aunque es importante destacar que algunos instrumentos compuestos y una preforma de instrumento bifacial (Figura 2B) registran operaciones de façonnage. Por otro lado, los soportes obtenidos de lascas cortas y anchas también presentan evidencia de una percusión directa blanda en su extracción. Dichas lascas fueron utilizadas para la confección de raspadores, artefactos con retoques sumarios y muescas. La mayoría de estos instrumentos presentaba retoques y microrretoques marginales.

Con relación a los desechos de talla $(81,3 \%$; Material suplementario 1) y a su estado, prevalecen las lascas fracturadas sin talón $(52,1 \%)$, seguidas 


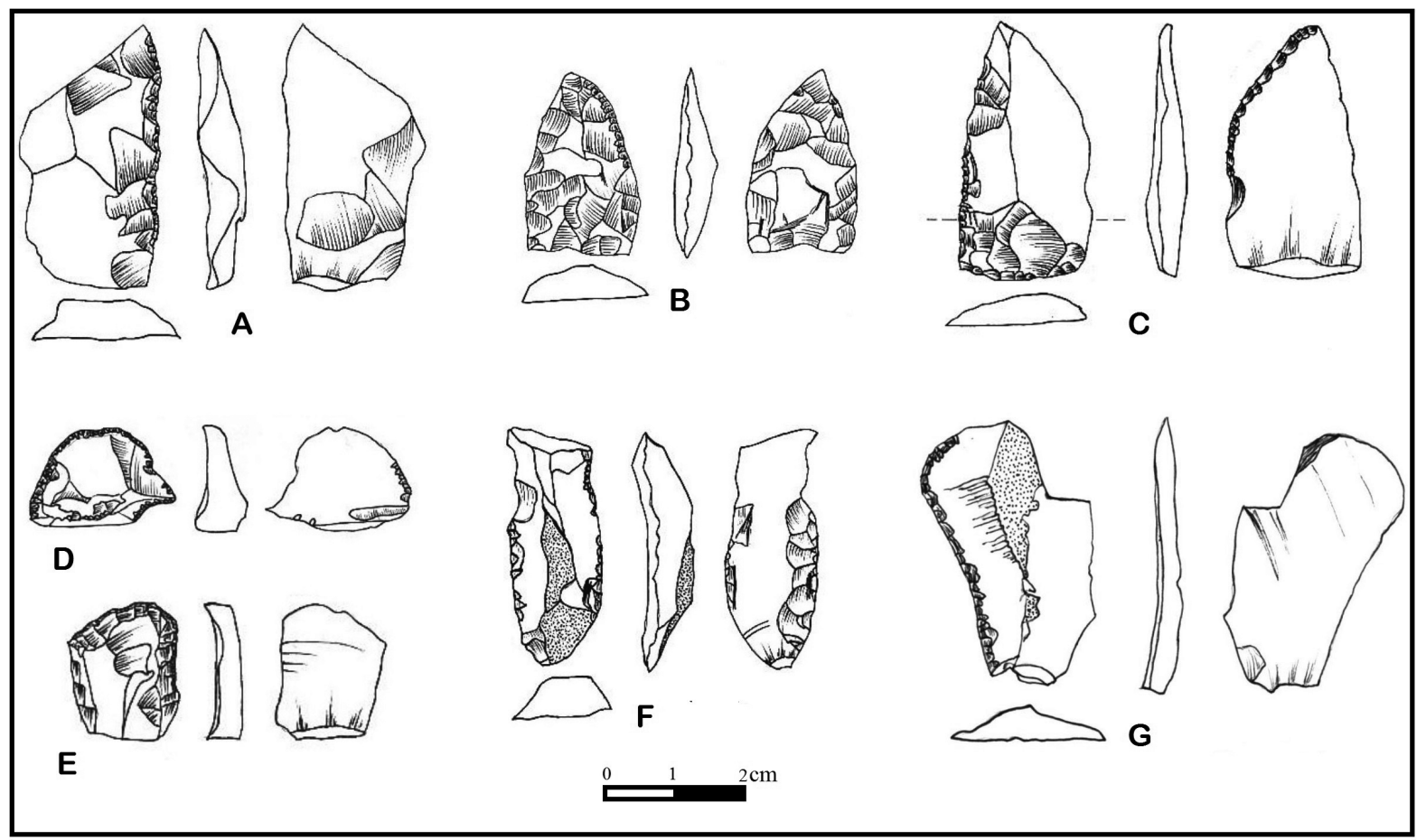

Referencias: A- Instrumento compuesto de ortocuarcita GSB (FCS.LLA.84); B- Preforma de punta de ortocuarcita GSB (FCS.LLA.268); CRaedera de ortocuarcita GSB (FCS.LLA.101); D- Instrumento compuesto de ftanita (FCS.LLA.438); E- Raspador de ftanita (FCS.LLA.213); F- Instrumento compuesto de ftanita (FCS.LLA.414); G- Cuchillo de limolita silicificada (FCS.LLA.552).

Figura 2. Instrumentos tallados del sitio LLA.

por las enteras $(27,2 \%)$ y las fracturadas con talón $(20,7 \%)$. Los tamaños más frecuentes son el pequeño $(58,7 \%)$ y el mediano pequeño $(32,6 \%)$, y los módulos predominantes son el corto ancho $(39,2 \%)$ y el mediano normal (28,2\%). Los tipos de lascas más representados en esta roca son los angulares $(30,4 \%)$ y los de adelgazamiento $(30,4 \%)$, seguidos por los indeterminados $(17,4 \%)$ y de reactivación de instrumentos $(10,9 \%)$, entre otros. Finalmente, los tipos de talones más frecuentes son los lisos (79\%) y los filiformes (12,3\%).

\section{Ftanita}

En el sitio se recuperaron cinco núcleos de ftanita, tres de los cuales están enteros, y los dos restantes, fragmentados. La forma en que esta materia prima se presenta en las zonas de abastecimiento y la presencia de remanentes de corteza en diferentes caras de los núcleos permitió inferir que los soportes provienen de nódulos. Entre los enteros, uno es piramidal irregular (Figura 3A) con lascados bidireccionales (tamaño pequeño y módulo corto ancho), y los dos restantes, indeterminados (Figura 3C y D). De las dos plataformas de percusión en el núcleo piramidal, una es plana y se encuentra agotada, mientras que la otra está parcialmente agotada. Uno de los núcleos indeterminados es mediano grande, de módulo mediano normal, y presenta lascados bidireccionales y dos plataformas de percusión parcialmente agotadas (Figura 3C); mientras que el restante es grande, de módulo corto ancho, registra lascados multidireccionales y las tres plataformas están agotadas (Figura 3D). Por último, entre los fragmentados se encuentra un núcleo bipolar con corteza (Figura 3B).

El $32,3 \%$ de los instrumentos del sitio se confeccionó sobre ftanita; el $77,1 \%$ se encuentra en estado entero, y el 22,9\% restante, fracturado. Los raspadores conforman el grupo tipológico más representado (58,1\%; Figura 2E) y, en proporciones inferiores, se encuentran los fragmentos de instrumentos indeterminados $(12,8 \%)$, los instrumentos compuestos (Figura 2D y F) y las puntas de proyectil (9,7\% cada uno), los instrumentos bifaciales $(6,5 \%)$ y un artefacto con retoque sumario (Material suplementario 2). Los soportes seleccionados son lascas indeterminables $(60 \%)$, seguidas por angulares y formas base indeterminables $(14,5 \%$ cada una), lascas externas $(8,6 \%)$ y lascas internas bipolares $(2,8 \%)$. Con relación al tamaño de los instrumentos enteros, el mayormente representado es el pequeño $(62,9 \%)$ al que le sigue el mediano pequeño (25,9\%, Material suplementario 3). En cuanto al módulo de longitud 


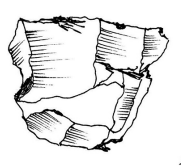

A
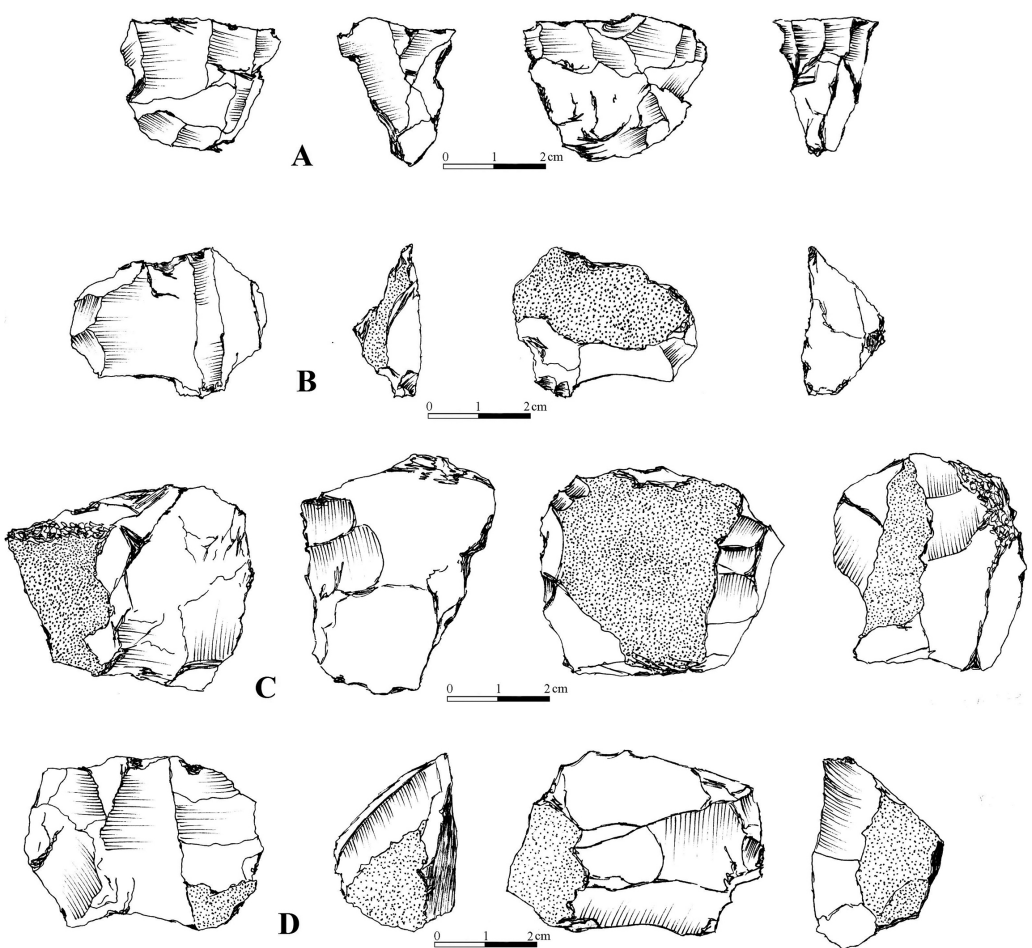

Referencias: A- Núcleo piramidal irregular (FCS.LLA.250); B- Fragmento de núcleo bipolar con lascados bidireccionales (FCS.LLA.244); C y D- Núcleos enteros indeterminados (FCS.LLA.279 y FCS.LLA.98).

Figura 3. Núcleos de ftanita del sitio LLA.

y anchura, prevalece el mediano normal (44,5\%; Material suplementario 4).

Con respecto al modo de talla, solo se utilizaron operaciones de façonnage en la elaboración de un instrumento bifacial y en las puntas de proyectil. Para estos se aplicaron retoques extendidos y microrretoques marginales. En la manufactura de raspadores (Figura 2E) y artefactos de retoque sumario se utilizaron mayormente retoques y microrretoques unifaciales marginales.

Con relación a los desechos de talla $(68,4 \%$; Material suplementario 1), predominan las lascas fracturadas sin talón $(58,5 \%)$, seguidas por las enteras $(30,2 \%)$ y las fracturadas con talón $(11,3 \%)$. El tamaño más frecuente es el pequeño $(62,5 \%)$, y entre los módulos predominan el mediano normal $(43,8 \%)$ y el corto ancho $(31,3 \%)$. La lasca más representada es la angular (50\%), seguida por las de adelgazamiento, secundarias y de reactivación de instrumentos (12,5\% cada una). Los talones lisos $(77,3 \%)$ fueron los más representados.

\section{Metacuarcita}

En esta materia prima se registraron cuatro percutores y tres instrumentos de molienda (Material suplementario 2). Entre los primeros, uno se encuentra entero y los tres restantes, fracturados; mientras que entre los segundos, todos se hallan fracturados. El rodado es la forma base de todos los percutores; en dos de ellos se encontraron estrías y piquetes machacados sobre las aristas, lo cual se vincula con las actividades de percusión. El tamaño del único percutor entero es muy grande y su módulo es corto ancho (Material suplementario 3 y 4). Los soportes utilizados para la confección de los instrumentos de molienda -en cuyas superficies se evidencia un tratamiento de picado y pulidoson indeterminables. Con respecto a los desechos de talla (Material suplementario 1), estos se encuentran representados por tres lascas fracturadas sin talón, una lasca indeterminable entera y cinco desechos indeterminables.

\section{Limolita silicificada}

Los instrumentos en esta materia prima están representados por dos cuchillos unifaciales enteros de filo lateral y un percutor con la presencia de leves piquetes y estrías superficiales (Material suplementario 2). Es importante destacar que no se registraron operaciones de façonnage sobre esta materia prima. Para la confección de los cuchillos se seleccionaron lascas angulares como soportes, de espesor delgado en uno, y grueso en otro. La serie técnica utilizada fue la talla, retoque y microrretoque marginales para la manufactura de uno de los cuchillos; mientras que en el otro se observaron únicamente microrretoques marginales (Figura 2G). Los tamaños representados son mediano grande y grande, mientras que los módulos son mediano normal y corto muy ancho (Material suplementario 3 y 4 ). La forma base del artefacto con filo natural con rastros complementarios corresponde a una lasca indeterminable, mientras que la del percutor es un rodado.

Con respecto a los desechos de talla $(78,6 \%$; Material suplementario 1), prevalecen las lascas enteras $(60 \%)$ y las fracturadas sin talón $(40 \%)$. El tamaño predominante es el pequeño $(52 \%)$ y el 
módulo es corto anchísimo (40\%). Las lascas de dorso natural $(50 \%)$ son las más representativas y el tipo de talón con mayor frecuencia es el liso (84\%). Se destaca la presencia de remanente de corteza, tanto en los instrumentos como en los desechos de talla.

\section{Otras materias primas}

En muy baja proporción se identificaron desechos de talla e instrumentos en el resto de las materias primas (Material suplementario 1 y 2). En sílice se reconocieron dos raspadores frontales enteros, ambos elaborados sobre lascas angulares a partir de retoques extendidos y microrretoques marginales, un rodado utilizado como percutor, cuatro lascas fracturadas sin talón y una lasca angular entera. En granito se hallaron dos percutores sobre rodado y dos boleadoras, una lasca entera y otra fracturada sin talón. En riolita, chert silíceo, dacita y basalto solo se identificaron lascas (angulares, de arista, de adelgazamiento, bipolar e indeterminables), mientras que en arenisca se reconoció un alisador/ pulidor entero; en cuarzo, un percutor y tres desechos indeterminables; en esquisto, dos fragmentos de instrumentos de molienda; en cuarcita sacarosa, dos desechos indeterminables; y en xilópalo, una lasca de arista con rastro complementario y reserva de corteza.

\section{DISCUSIÓN}

En el sitio LLA se registra una amplia variabilidad de materias primas líticas y del tratamiento que recibió cada una de ellas. Entre las rocas talladas, las más representadas son la ortocuarcita GSB $(54,4 \%)$, procedente del centro-sur del Sistema Serrano de Tandilia (Flegenheimer et al., 1996; Colombo, 2011), y la ftanita $(24,4 \%)$ proveniente del mismo sector serrano o de la porción noroccidental (Messineo y Barros, 2015). Esta tendencia se condice con la registrada por Barros (2001) y Hoguin y March (2008) para otros sitios lagunares de las cuencas superior e inferior del río Quequén Salado (e.g., Laguna Tres Reyes, Puente Oriente, Cueva del Tigre del Norte y La Represa). Una posible explicación de por qué se prefirieron estas rocas, ubicadas a distancias mayores del sitio por sobre las locales (e.g., limolita silicificada), podría vincularse con su calidad para la talla, la forma en que se presentan en las canteras (nódulos de diferentes tamaños en ftanita y bloques en ortocuarcita) y los objetivos de talla buscados (instrumentos versátiles en ortocuarcita). Por su parte, en proporciones inferiores al $5 \%$ se registran la limolita silicificada, presente en afloramientos cercanos al sitio (Madrid y Salemme, 1991), la riolita del Sistema Serrano de Ventania (Oliva y Moirano, 1997), la sílice, la dacita, el xilópalo y el basalto procedentes de rodados costeros (Bonomo, 2005) y el chert silíceo que se encuentra en afloramientos primarios y secundarios de la subregión Pampa Seca (entre 460 y $600 \mathrm{~km}$ al oeste del sitio) (Carrera Aizpitarte et al., 2015). En referencia a las rocas no talladas, se registraron cuarzo, granito, arenisca, esquisto y metacuarcita procedentes del Sistema Serrano de Ventania y de los cursos fluviales que nacen de este sistema serrano (Bayón et al. 2006; Catella, 2014).

Las cadenas operativas de ortocuarcita GSB (Figura 4) no tienen representadas las primeras etapas, vinculadas al descortezamiento y reducción inicial de los núcleos, lo cual implicaría que tales actividades se habrían Ilevado a cabo en las canteras que se localizan en las sierras de Tandilia. Los materiales líticos hallados en las inmediaciones de los afloramientos (e.g., nódulos testeados, desechos indeterminables, lascas de descortezamiento, núcleos de diferentes morfologías) sugieren un descortezamiento inicial en estos sitios, que involucra una cuota de tiempo y esfuerzo invertidos en el abastecimiento (Paulides, 2006; Colombo, 2011). Varios investigadores han planteado que para algunos sectores del área Interserrana, el abastecimiento de esta roca se habría dado directamente por medio de viajes logísticos (Gould y Saggers, 1985) hacia las canteras por parte de un grupo de artesanos y aprendices que tenían un amplio conocimiento de las técnicas de talla y del lugar, lo cual permitió un aprovechamiento óptimo de la materia prima en los sitios alejados de las fuentes (Bayón y Flegenheimer, 2004; Colombo, 2011). Con relación a este punto, es importante destacar que Bayón y Flegenheimer (2004) propusieron que, durante el Holoceno tardío en la región pampeana, el acceso a las canteras pudo estar más restringido, con lo cual los grupos cazadores-recolectores debieron intensificar las redes sociales de interacción para poder acceder a diversos recursos.

Los soportes obtenidos en las canteras-taller fueron trasladados e ingresaron al sitio en un estado avanzado de reducción o como artefactos ya formatizados. Tal propuesta está sustentada a partir del predominio de lascas internas, un escaso porcentaje 
de corteza y la ausencia de núcleos. En cuanto a los objetivos de talla, se evidencia la búsqueda de formas base alargadas para confeccionar instrumentos compuestos, raederas e instrumentos bifaciales y soportes cortos y anchos para elaborar raspadores e instrumentos con retoques sumarios (Figura 4).

Algunos autores proponen que la ortocuarcita GSB fue seleccionada especialmente para confeccionar instrumentos destinados a múltiples actividades, los cuales se elaboraron a partir de soportes grandes de tendencia laminar, ya que presentan una mayor resistencia de los filos al uso (Leipus y Mansur, 2007; Massigoge y Pal, 2011; Pal, 2012). En el sitio LLA se observa la misma tendencia, lo que evidencia una significativa inversión de trabajo en la manufactura de instrumentos a partir del registro de un porcentaje elevado de retoques y microrretoques extendidos, de la utilización de diversas técnicas de adelgazamiento y de la estandarización de las formas base (lascas grandes y módulos alargados para confeccionar filos largos). El empleo de estas técnicas requirió, durante el proceso de talla, de un mayor conocimiento práctico (savoir-faire) y de una cierta destreza manual, que reflejaban las habilidades particulares del tallador (Pelegrin, 1991). Esta misma tendencia fue registrada en diversos contextos arqueológicos de la subregión Pampa Húmeda, como en los sitios Laguna Tres Reyes 1, Laguna Ovalla y La Represa, localizados en la cuenca del río Quequén Salado (Barros, 2001; Hoguin y March, 2008); Cortaderas y Las Brusquillas 1, en el sector meridional del área Interserrana (Massigoge y Pal, 2011); y Calera y El Puente, en el sector noroccidental de Tandilia (Pal, 2012). En esta materia prima se registró la utilización de técnicas de talla bipolar, situación que también fue observada en otros contextos del río Quequén Salado (Barros, 2001; March et al., 2011). Se plantea que el uso de esta técnica fue una forma de aprovechamiento intensivo de rocas de buena calidad, ya que permitió explotar al máximo pequeños volúmenes de materias primas que no podrían haber sido utilizados de otra forma.

Las cadenas operativas mayormente desarrolladas y completas en el sitio corresponden a la ftanita (Figura 4), ya que en esta materia prima se encuentran representados los únicos núcleos del sitio (Figura 3). La distancia a las fuentes de esta materia prima, la alta frecuencia de esta roca, la presencia de núcleos con remanentes de corteza y la variabilidad en los grupos tipológicos de los instrumentos

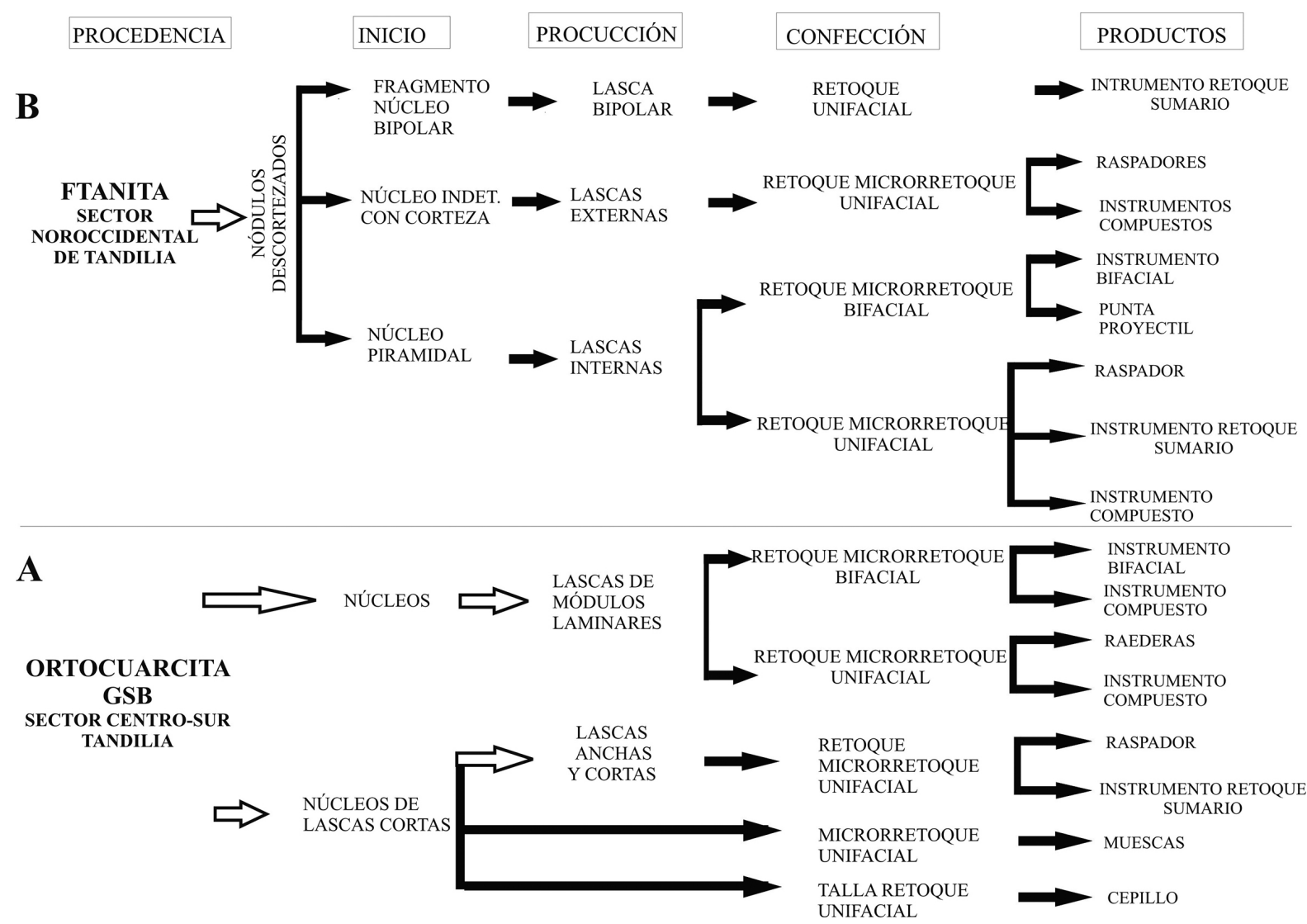

Referencias: Flechas blancas significan actividades ausentes en el sitio, y flechas negras, actividades desarrolladas en el sitio.

Figura 4. Cadenas operativas de ortocuarcita GSB y ftanita. 
permiten inferir un abastecimiento directo mediante partidas logísticas (Gould y Saggers, 1985) desde la laguna a los afloramientos del Sistema Serrano de Tandilia. El escaso registro de lascas externas y la presencia poco significativa de corteza en LLA evidenciarían que las etapas iniciales del proceso de reducción no se habrían efectuado en el lugar.

Los datos permiten identificar algunas etapas intermedias en el sitio, es decir, una posible obtención primaria de nódulos en las fuentes, los cuales ingresaron a aquel bajo la forma de núcleos parcialmente descortezados. Una vez allí, uno de los objetivos fundamentales en la ftanita fue la producción de soportes poco estandarizados y con escasa modificación (e.g., lascas internas de diferentes tamaños y módulos), sobre los cuales se confeccionaron una amplia variedad de instrumentos, entre los que se destacan los raspadores pequeños (Figura 2E). La selección de esta roca para la elaboración de raspadores también fue registrada en diversos contextos arqueológicos del área Interserrana (Bayón et al., 2006; Leipus, 2006; Massigoge y Pal, 2011; Pal, 2012; Barros et al., 2015), lo cual podría indicar una mejor adecuación de esta materia prima para tal fin. En menor medida, se destinaron soportes para la confección de puntas de proyectil e instrumentos compuestos (Figura 2D). Por último, cabe destacar la maximización en el uso de esta materia prima, que se evidencia en el registro de la talla bipolar, el reducido tamaño e indeterminación de los tipos de núcleos y el pequeño tamaño de los instrumentos.

La reconstrucción de la cadena operativa de la limolita silicificada es incompleta y solo se encuentra representada la obtención, mediante la percusión directa, de lascas grandes con corteza para confeccionar cuchillos y la manutención de dichos instrumentos (Figura 5). Además, se puede inferir que algunas actividades vinculadas con el desbaste pudieron llevarse a cabo en el sitio, aunque no se registraron núcleos en él. El abastecimiento de esta roca pudo darse de dos maneras posibles: ya sea a partir de visitas logísticas a las canteras para proveerse de esta materia prima o mediante un aprovisionamiento efectuado en el marco de otras actividades (Binford, 1979), teniendo en cuenta que la fuente de esta roca está próxima a la laguna. El ingreso de la roca a LLA pudo darse bajo la forma de grandes lascas secundarias o como núcleos medianamente descortezados. En este punto, es importante destacar que, al ser esta roca la más cercana al sitio, su utilización fue limitada, ya que solo se hallaron tres instrumentos y escasos desechos de talla. Posiblemente los grupos priorizaron rocas de excelente calidad para la talla, aunque estas se encontraran a distancias mayores de la laguna. Esta misma tendencia se registra para otros sitios de las cuencas superior e inferior del río Quequén Salado (Barros, 2001; Madrid et al., 2002, entre otros).

En cuanto a los rodados costeros (sílice, dacita, basalto y xilópalo), las cadenas operativas se presentan fragmentadas (Figura 5), y el ingreso de aquellos pudo darse de diversos modos, ya sea en su forma original o como instrumento. Los grupos cazadores-recolectores que ocuparon LLA no priorizaron la explotación de este recurso lítico, cuyo aprovisionamiento habría sido esporádico, es decir, como una actividad secundaria dentro de la búsqueda de otros tipos de recursos costeros (Binford, 1979; ver otros casos en Bonomo, 2005). Esto podría deberse a la incompatibilidad entre el tamaño de los rodados y los soportes buscados para la manufactura de ciertos instrumentos (Leipus, 2006; Pal, 2012). En la reducción de los rodados de sílice se utilizó la técnica de percusión directa para la obtención de los soportes y la manufactura de raspadores. Para el resto de los rodados se empleó la talla bipolar, la cual permitió una fácil manipulación y aprovechamiento de estas rocas, situación que fue constatada en otros sitios del río Quequén Salado (e.g., Laguna Ovalla y Tres Reyes 1) (Barros, 2001).

Para las escasas rocas procedentes del Sistema Serrano de Ventania (e.g., metacuarcita, riolita, granito y arenisca), localizadas a una distancia de más de 100 km de la laguna, es difícil identificar cómo fue el abastecimiento por parte de los talladores, aunque posiblemente haya sido de forma directa, ya sea mediante partidas logísticas hacia las canteras o durante los movimientos de los campamentos residenciales durante el ciclo de movilidad anual de estos grupos. Esta escasa utilización de las rocas de Ventania también se registra en los conjuntos líticos de otros sitios de las cuencas superior e inferior del río Quequén Salado (Madrid et al. 2002). En cuanto a la riolita, se evidencia una cadena operativa incompleta (Figura 5), que refleja la búsqueda de lascas como soportes, con técnicas de percusión directa blanda en la extracción de estas (preparación de talones). Por su parte, en metacuarcita, granito y arenisca se identificaron instrumentos manufacturados mediante técnicas de picado y pulido de la superficie. Los artefactos de molienda habrían servido para realizar actividades 
de procesamiento de diferentes productos (e.g., vegetales, minerales, etc.), mientras que las boleadoras habrían sido utilizadas como armas para la caza de diversas especies animales. Estos instrumentos presentan cadenas incompletas, ya que no se evidencian las actividades vinculadas con su producción. Asimismo, se destaca la presencia de artefactos modificados por uso, como los percutores, para los cuales se seleccionaron nódulos procedentes de los valles fluviales que descienden de las Sierras de Ventania. Posiblemente, estos elementos y parte de los artefactos de molienda son instrumentos que se dejaron equipando los lugares (sensu Khun, 1994).

Por último, en el chert silíceo (materia prima extraareal) no se registra ninguna de las etapas de manufactura de instrumentos y se encuentra esta únicamente representada por una lasca de arista sin corteza (Figura 5). Aunque es difícil inferir el modo de abastecimiento de esta roca a partir de este registro en LLA, es importante mencionar que en diversos contextos de la subregión Pampa Húmeda, la presencia de litologías extraareales se vinculó a un modo de adquisición indirecto, mediante redes de interacción que conectaron a los grupos cazadores-recolectores que ocupaban distintas áreas de la región pampeana durante el Holoceno tardío (Bonomo, 2005; Bayón et al., 2006; Mazzanti, 2006; Messineo, 2011).

\section{CONCLUSIONES}

En el sitio LLA se observa una amplia variabilidad de materias primas líticas explotadas, procedentes de diversos sectores de la región pampeana (sistemas serranos de Tandilia y Ventania, costa atlántica, afloramientos aislados del área Interserrana y la subregión Pampa Seca). Los cazadores-recolectores que habitaron la laguna priorizaron la calidad y el volumen de los nódulos o núcleos de rocas localizadas a distancias mayores (e.g., ortocuarcita GSB y ftanita) a partir de la implementación de una movilidad logística que habría permitido un abastecimiento directo de dicha roca. En menor medida, se explotaron de forma directa rocas locales (e.g., limolita silicificada) y fuentes que se encuentran más cercanas al sitio (e.g., rodados costeros). La ortocuarcita GSB evidenció un uso estandarizado para la confección de instrumentos con soportes laminares y filos largos (e.g., raederas e instrumentos compuestos) y de otros tipos de instrumentos de menor tamaño. En ftanita se registró la producción de soportes poco estandarizados (e.g., lascas internas de diferentes tamaños y módulos, algunas externas) sobre las cuales se confeccionaron una amplia variedad de instrumentos, entre los que se destacan los raspadores de tamaño pequeño. Esta evidencia, similar a otros sitios de la cuenca y de las áreas Interserrana y Tandilia, permite identificar

\section{\begin{tabular}{l|l|l|l|}
\hline PROCEDENCIA & INICIO & PRODUCCIÓN & PONFECCIÓN
\end{tabular}}
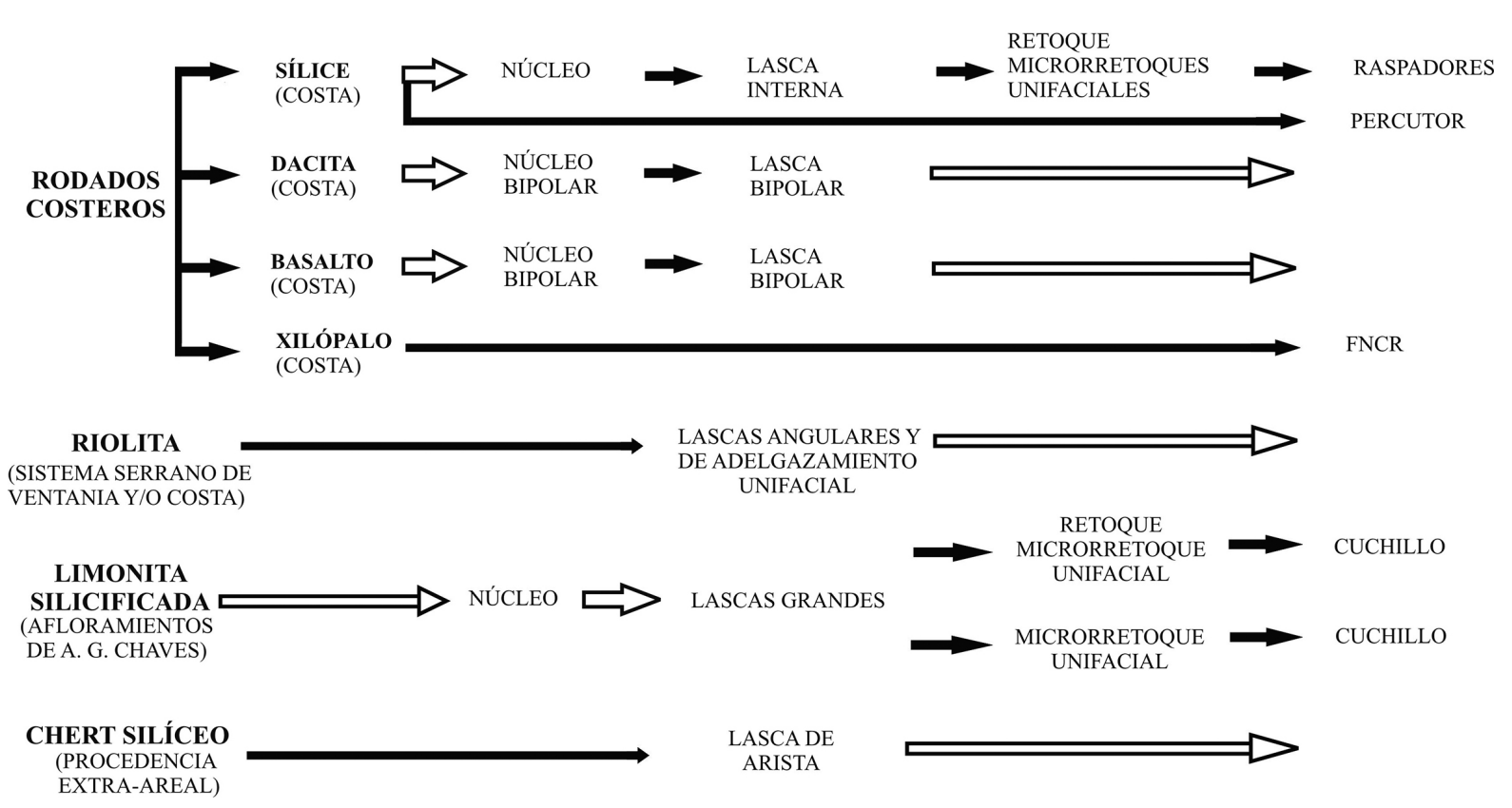

Referencias: Flechas blancas significan actividades ausentes en el sitio, y flechas negras, actividades desarrolladas en el sitio.

Figura 5. Cadenas operativas de rodados costeros, limolita silicificada, riolita y chert silíceo. 
ciertas tendencias en común en cuanto al modo de producción (formas buscadas para cada materia prima, morfología y tamaño de las piezas), que hace referencia a un conocimiento socialmente compartido de un saber hacer por parte de los talladores y usuarios de la tecnología lítica durante el Holoceno tardío. Esta diversidad en el uso y en el tratamiento de cada una de las rocas podría estar relacionada con las distancias a las diversas fuentes de abastecimiento, las cualidades de las rocas para los productos buscados, los circuitos de movilidad y las redes sociales de interacción de los grupos humanos, entre otros factores.

\section{Agradecimientos}

Agradezco al Dr. Pablo Messineo por la confianza y el apoyo brindado en la lectura y edición del presente trabajo. También quiero brindar mi gratitud a las Dras. María P. Barros y Florencia Santos Valero por la lectura del manuscrito y sus valiosos aportes para mejorarlo. A los evaluadores, por las sugerencias y correcciones que mejoraron sustancialmente el trabajo.

\section{REFERENCIAS CITADAS}

Aschero, C. (1975). Ensayo para una clasificación morfológica de artefactos líticos. Informe al CONICET. MS.

Aschero, C. (1983). Ensayo para una clasificación morfológica de artefactos líticos. Revisión. Guías de Cátedra. Ergología y Tecnología. Facultad de Filosofía y Letras, Universidad de Buenos Aires. MS.

Aschero, C. y Hocsman, S. (2004). Revisando cuestiones tipológicas en torno a la clasificación de artefactos bifaciales. En M. Ramos, A. Acosta y D. Loponte (Comps.), Temas de Arqueología. Análisis lítico (pp. 7-25). Universidad Nacional de Luján.

Barros, M. P. (2001). Análisis del material lítico de superficie de la región del Río Quequén Salado (Holoceno medio y tardío), Provincia de Buenos Aires [Diplôme d'études approfondies. Universidad de París I, Panthéon-Sorbonne].

Barros, M. P., Messineo, P. G. y Colantonio, M. J. (2015). Chert quarries and workshops in the Humid Pampa sub-region: New contributions on exploitation techniques and circulation through study of chaînes opératoires. Quaternary International, 375, 99-112.

Bayón, C. y Flegenheimer, N. (2004). Cambio de planes a través del tiempo para el traslado de rocas en La Pampa bonaerense. Estudios Atacameños, 28, 59-70.
Bayón, C., Flegenheimer, N. y Pupio, A. (2006). Planes sociales en el abastecimiento y traslado de roca en la Pampa Bonaerense en el Holoceno Temprano y Tardío. Relaciones de la Sociedad Argentina de Antropología, $31,19-45$.

Bellelli, C., Guráieb, A. G. y García, J. A. (1985-1987). Propuesta para el análisis y procesamiento por computadora de desechos de talla lítica (DELCO Desechos de talla líticos computarizados). Arqueología Contemporánea, 2(1), 36-53.

Binford, L. R. (1979). Organization and formation processes: looking at curated technologies. Journal of Anthropological Research, 35, 255-273.

Böeda, E. (1993). Le débitage discoïde et le débitage Levallois récurrent centripède. Bulletin de la Société Préhistorique Française, 90(6), 392-404.

Bonnat, G. F. (2021). Análisis de la organización de la tecnología lítica de los grupos cazadores-recolectores tempranos del área de Tandilia oriental, Buenos Aires. Sociedad Argentina de Antropología.

Bonomo, M. (2005). Costeando las Ilanuras. Arqueología del litoral marítimo pampeano. Sociedad Argentina de Antropología.

Carrera Aizpitarte, M., Berón, M. y Blasi, A. (2015). Study of siliceous outcrops of Meseta del Fresco, La Pampa, Argentina. Quaternary International, 375, 27-43.

Catella, L. (2014). Movilidad y utilización del ambiente en poblaciones cazadoras-recolectoras del sur de la Región Pampeana: la Cuenca del Arroyo Chasicó como caso de estudio [tesis doctoral inédita. Universidad Nacional de La Plata].

Colombo, M. (2011). El área de abastecimiento de las ortocuarcitas del grupo Sierras Bayas y las posibles técnicas para su obtención entre los cazadores y recolectores pampeanos. Intersecciones en Antropología, 12, 155-166.

Flegenheimer, N., Kain, S., Zárate, M. y Barna, A. (1996). Aprovisionamiento de rocas cuarcíticas en Tandilia. Las canteras del Arroyo Diamante. Arqueología, 6, 117-143.

Gould, R. y Saggers, S. (1985). Lithic procurement in Central Australia. A closer look at Binford's idea of embededness in archaeology. American Antiquity, 50, 117-136.

Hoguin R. y March, R. (2008). Una aproximación al análisis tipo-tecnológico de los artefactos líticos del sitio La Represa (curso inferior del Quequén Salado, pcia. de Buenos Aires). Arqueología, 14, 103-136. 
Inizan, M., Reduron, M., Roche, H. y Tixier, J. (1995). Technologie de la Pierre Taillée. CREP.

Kuhn, S. (1994). A formal approach to the design and assembly of mobile toolkits. American Antiquity, 59(2), 426-442.

Leipus, M. (2006). Análisis de los modos de uso prehispánicos de las materias primas líticas en el Sudeste de la Región Pampeana. Una aproximación funcional [tesis doctoral inédita. Universidad Nacional de La Plata].

Leipus, M. y Mansur, M. E. (2007). El análisis funcional de base microscópica aplicado a materiales heterogéneos. Perspectivas metodológicas para el estudio de las cuarcitas de la Región Pampeana. En C. Bayón, A. Pupio, M. I. González, N. Flegenheimer y M. Frère (Eds.), Arqueología en las Pampas, Tomo 1 (pp. 179200). Sociedad Argentina de Antropología.

Lemonnier, P. (1992). Elements for Anthropology of Technology. Museum of Anthropology.

Leroi-Gourhan, A. (1943). Evolution et Techniques: L'Homme et la Matiere. Albin Michel.

Madrid, P., Politis, G., March, R. y Bonomo, M. (2002). Arqueología microrregional en el sudeste de la Región Pampeana Argentina: el curso del río Quequén Salado. Relaciones de la Sociedad Argentina de Antropología, 27, 327-355.

Madrid, P. y Salemme, M. (1991). La ocupación tardía del sitio 1 de la Laguna Tres Reyes, Adolfo González Cháves, Provincia de Buenos Aires. Boletín del Centro, 3, 165-179.

March, R., Rodríguez Loredo, C., Hoguin, R., Foisset, S., Joly, D. y Lucquin, A. (2011). Primeros resultados de la excavación del sitio de cazadores recolectores de La Represa. Oriente, partido de Coronel Dorrego, provincia de Buenos Aires, Argentina. Arqueología Rosarina Hoy, 3, 9-74.

Martínez, G. y Mackie, Q. (2003-2004). Late Holocene human occupation of the Quequén Grande River valley botton: settlement systems and an example of a built environment in the Argentine Pampas. Before Farming, 4, 178-202.

Massigoge, A. y Pal, N. (2011). Producción y uso de artefactos líticos en contextos cazadores-recolectores del Holoceno tardío del área Interserrana (Argentina): análisis integral de la diversidad tecno-morfológica y funcional. Revista Española de Antropología Americana, 41(1), 51-73.

Mazzanti, D. (2006). La constitución de territorios sociales durante el Holoceno tardío. El caso de las sierras de Tandilia, Argentina. Relaciones de la Sociedad Argentina de Antropología, 31, 277-300.
Messineo, P. G. (2008). Investigaciones arqueológicas en la cuenca superior del Arroyo Tapalqué (partidos de Olavarría y Benito Juárez, provincia de Buenos Aires) [tesis doctoral inédita. Universidad Nacional de La Plata].

Messineo, P. G. (2011). Investigaciones arqueológicas en la cuenca superior del Arroyo Tapalqué. Un modelo de ocupación humana para el centro de la subregión Pampa Húmeda durante el Holoceno tardío. Intersecciones en Antropología, 12, 275-291.

Messineo, P. G. y Barros, M. P. (2015). Lithic raw materials and modes of exploitation in quarries and workshops from the center of the Pampean grassland of Argentina. Lithic Technology, 40(1), 3-20.

Oliva, F. y Moirano, J. (1997). Primer informe sobre aprovisionamiento primario riolita en Sierra de la Ventana (Pcia. Buenos Aires, Argentina). En M. Berón y G. Politis (Eds.), Arqueología Pampeana en la década de los '90 (pp. 137-146). Museo de Historia Natural de San Rafael e Investigaciones Arqueológicas y Paleontológicas del Cuaternario Pampeano (INCUAPA), Facultad de Ciencias Sociales (UNICEN).

Pal, N. (2012). Tendencias temporales en las estrategias y uso de los materiales líticos recuperados en la cuenca superior del Arroyo Tapalqué (partido de Benito Juárez y Olavarría): Una perspectiva desde el análisis funcional [tesis doctoral inédita. Universidad Nacional del Centro de la Provincia de Buenos Aires].

Paulides, L. (2006). El núcleo de la cuestión. El análisis de los núcleos en los conjuntos líticos. En C. Pérez de Micou (Ed.), El modo de hacer las cosas. Artefactos y ecofactos en arqueología (pp. 67-97). Facultad de Filosofía y Letras, Universidad Nacional de Buenos Aires.

Pelegrin, J. (1991). Les savoir faire, une très longue histoire. Terrain, 16, 106-113

Politis, G. y Madrid, P. (1988). Un hueso duro de roer: análisis preliminar de la tafonomía del sitio Tres Reyes 1 (Pdo. de Adolfo González Chaves, Provincia de Buenos Aires). En N. Ratto y A. Haber (Eds.), De procesos, contextos y otros huesos (pp. 29-44). Universidad de Buenos Aires.

\section{Material suplementario}

Material suplementario 1. Categorías artefactuales del conjunto lítico del sitio LLA.

Material suplementario 2. Grupos tipológicos de los instrumentos por materia prima del sitio LLA.

Material suplementario 3. Tamaños de los instrumentos enteros por materia prima del sitio LLA.

Material suplementario 4. Módulos de L/A de los instrumentos enteros por materia prima del sitio LLA. 
